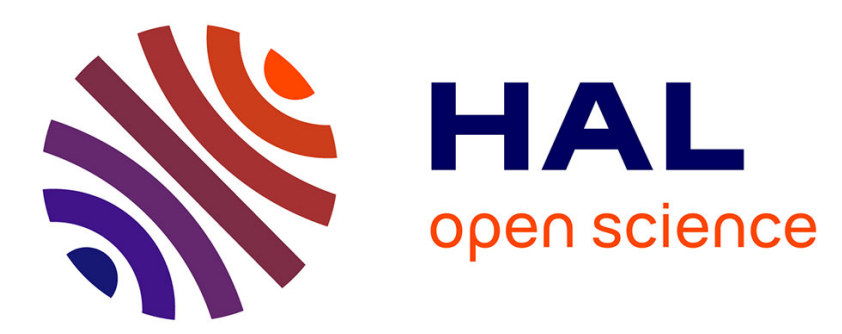

\title{
Gyrokinetic simulations of neoclassical transport using a minimal collision operator
}

Guilhem Dif-Pradalier, Virginie Grandgirard, Yanick Sarazin, Xavier Garbet, Philippe Ghendrih, P Angelino

\section{- To cite this version:}

Guilhem Dif-Pradalier, Virginie Grandgirard, Yanick Sarazin, Xavier Garbet, Philippe Ghendrih, et al.. Gyrokinetic simulations of neoclassical transport using a minimal collision operator. AIP Conference Proceedings, 2008, pp.53 - 63. 10.1063/1.3033731 . cea-01468364

HAL Id: cea-01468364 https://hal-cea.archives-ouvertes.fr/cea-01468364

Submitted on 15 Feb 2017

HAL is a multi-disciplinary open access archive for the deposit and dissemination of scientific research documents, whether they are published or not. The documents may come from teaching and research institutions in France or abroad, or from public or private research centers.
L'archive ouverte pluridisciplinaire HAL, est destinée au dépôt et à la diffusion de documents scientifiques de niveau recherche, publiés ou non, émanant des établissements d'enseignement et de recherche français ou étrangers, des laboratoires publics ou privés. 


\title{
Gyrokinetic simulations of neoclassical transport using a minimal collision operator
}

\author{
G. Dif-Pradalier, V. Grandgirard, Y. Sarazin, X. Garbet, Ph. Ghendrih and \\ P. Angelino
}

Association Euratom-CEA, CEA/IRFM, CEA-Cadarache, F-13108 St. Paul-lez-Durance cedex, France

\begin{abstract}
Conventional neoclassical predictions are successfully recovered within a gyrokinetic framework using a minimal Fokker-Planck collision operator. This operator is shown to accurately describe some essential features of neoclassical theory, namely the neoclassical transport, the poloidal rotation and the linear damping of axisymmetric flows while interestingly preserving a high numerical efficiency. Its form makes it especially adapted to Eulerian or Semi-Lagrangian schemes.
\end{abstract}

Keywords: Gyrokinetics, neoclassical theory

PACS: 52.35.Py, 52.35.Mw, 52.30.Cv

\section{INTRODUCTION}

Collisionless gyrokinetic theory [3] consensually provides today's deepest insight on turbulence-related problems in fusion plasma physics, especially allowing for a very accurate first-principle description of turbulent transport. On the other hand, the neoclassical theory was earlier coined to describe the effects of binary Coulomb collisions in an inhomogeneous magnetic field, when trapped particles are present [13]. As compared to the highly-collisional edge region of a tokamak, core conditions exhibit $(i)$ typical collision frequencies orders of magnitude smaller than the ones associated with the turblence and (ii) experimentally-measured transport levels, close to the turbulent transport levels and orders of magnitude larger than the predicted neoclassical ones. Thus, core plasmas are widely believed to be 'collisionless'.

Recent evidences from both the experimental side [4, 2, 6] and from gyrokinetic modelling [18] are contributing to modify this classical idea. The adjunction of neoclassical theory to gyrokinetic models is therefore of great current interest: the onset and control of the improved confinement regimes necessary to operate e.g. Iter require the access to advanced regimes where the turbulence can be locally suppressed. In such regimes, (i) neoclassical transport becomes dominant. Also, an increasing number of experimental observations [21] tends to emphasise the fundamental role of plasma rotation for the onset and the control of these improved regimes, a special focus being held on (ii) poloidal rotation. This latter quantity, even with turbulence, is widely believed to be set by neoclassical theory. The generation of axisymmetric (zonal) flows by turbulence, as recently emphasised, could however drastically modify this picture [19, 7]. These flows have been shown to survive the linear Landau damping process in the collisionless regime [22]. As a result, the level of transport can be underestimated when collisions are 
not taken into account [18].

The calculation of the collisional processes in a hot plasma is notoriously complex. The concept alone of collision in such a medium is rather subtle and since collisions between particles depend on their relative velocities, the overall collisional result is an integrated effect of non-local interactions between particles of all velocities. As a consequence, model operators which allow to recover the main neoclassical results while preserving some interesting simplicity are especially attractive. In this paper, we report the implementation of a such simplified operator in the global and full $-f$ gyrokinetic semi-Lagrangian GYSELA code and its successful confrontation against theoretical neoclassical predictions. The model equations are detailed in the following section, along with the adopted collision operator. While the accessible physical features with such a simplified operator are discussed in great detail subsequently, the last section displays a quantitative confrontation to conventional neoclassical predictions. We especially accurately recover the predicted neoclassical transport, the collisional zonal flow damping. We also report for the first time, to our knowledge, the reverse of the poloidal rotation with the collisionality regime, as predicted analytically.

\section{A MINIMAL COLLISION OPERATOR CONTAINING CONVENTIONAL NEOCLASSICAL THEORY}

The GYSELA code has been upgraded to perform gyrokinetic simulations of neoclassical transport using a simplified collision operator. A full Coulomb collisional transport has notoriously considerable implementation difficulties in Eulerian-like numerical schemes and a simplified version is therefore highly attractive, provided it keeps the important properties of the complete operator. The precise derivation of this simplified model can be found in a recent work [10] where it is especially shown that it embeds the exact neoclassical transport as would be calculated by a full Fokker-Planck operator while preserving some simplicity. The GYSELA code is based on a semi-Lagrangian scheme [11], applied to solve the gyrokinetic equation:

$$
\partial_{t} f+\left(\mathbf{v}_{E}+\mathbf{v}_{D}\right) \cdot \nabla f+v_{\|} \nabla_{\|} f+\mathrm{d}_{\mathrm{t}} \mathrm{v}_{\|} \partial_{\mathrm{v}_{\|}} \mathrm{f}=\mathscr{C}(\mathbf{f})
$$

The left-hand side of Eq.(1) is classically the collisionless gyrokinetic equation as discussed e.g. in Ref. [12], $f$ represents the full ion distribution function and $v_{\|}$the velocity along the magnetic field lines. Self-consistency is achieved for the electric field by solving the quasi-neutrality condition:

$$
\frac{e}{T_{e}(r)}\left[\phi-\phi_{00}\right]-\frac{1}{n_{e q}(r)} \nabla_{\perp} \cdot\left[\frac{n_{e q}(r)}{B_{0} \omega_{c i}} \nabla_{\perp} \phi\right]=\frac{2 \pi B}{m n_{e q}(r)} \int_{0}^{\infty} d \mu \int_{-\infty}^{+\infty} d v_{\|} \mathscr{J} \cdot\left[f-f_{\text {init }}\right]
$$

In the remainder of the paper, the electronic response is assumed to be adiabatic: $\delta n_{e} / n_{e q}=e\left(\phi-\phi_{00}\right) / T_{e}(r)$, where $\phi_{00} \equiv \iint d \theta d \varphi / 4 \pi^{2} \phi$ accounts for the flux-surface average of the electric potential $\phi$ which initially is a perturbation. The ion cyclotron pulsation is $\omega_{c}$ and $f_{\text {init }}$ refers to the initial ion distribution function. A simplified Lorentztype ion-ion collision operator $\mathscr{C}$ conserving the number of particles is implemented in 


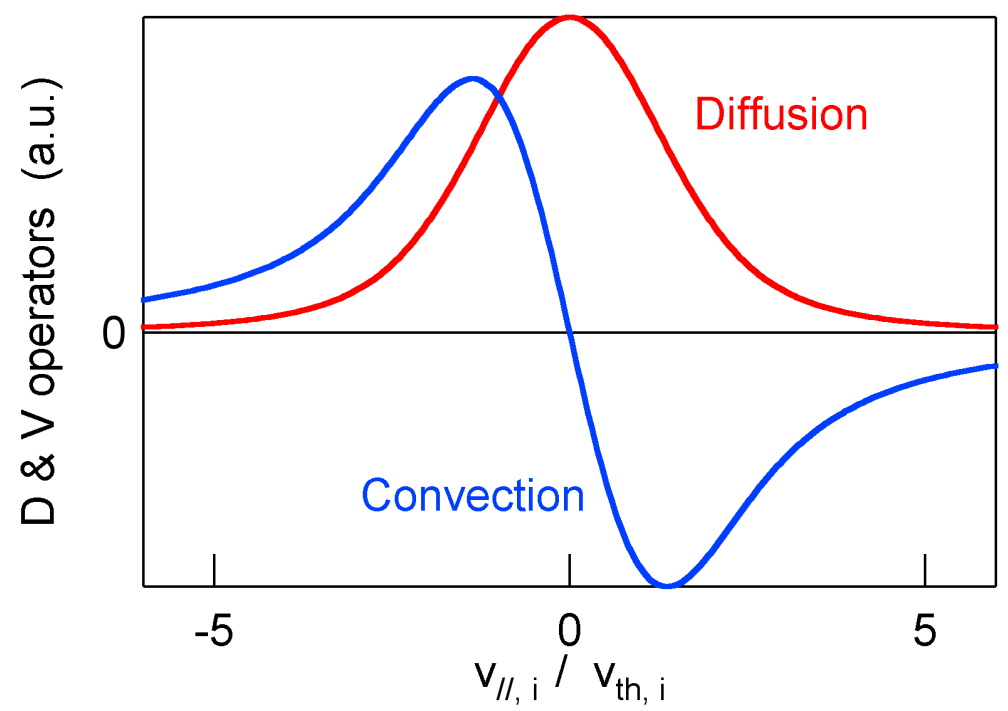

FIGURE 1. (Color online) Parallel velocity dependance of the diffusive and convective operators $\mathscr{D}$ and $\mathscr{V}$ in Eqs.(4) and (5).

the right-hand side of Eq.(1):

$$
\mathscr{C}=\partial_{v_{\|}}\left\{\mathscr{D} \partial_{v_{\|}} f-\mathscr{V} f\right\}
$$

which, as compared to electron-ion and electron-electron collisions, accounts for the dominant contribution to neoclassical heat transport. Operators $\mathscr{D}$ and $\mathscr{V}$ (see Fig. 1) respectively model a diffusion and a drag:

$$
\begin{aligned}
& \mathscr{D}=3 \frac{\sqrt{\pi}}{2} \frac{v_{T}^{3} \varepsilon^{3 / 2}}{q R_{0}} v_{\star} \frac{\Phi(v)-G(v)}{2 v} \\
& \mathscr{V}=-\frac{v_{\|}}{v_{T}^{2}} \mathscr{D}
\end{aligned}
$$

The first piece accounts for diffusion in parallel velocity $\mathscr{D}=\left\langle\Delta v_{\|}^{2}\right\rangle / 2$ generated by the Coulomb binary interactions. It is essentially responsible for the neoclassical diffusive transport and models the resonant enhancement of collisional effects in the presence of local trapping. The second piece is often referred to as 'dynamic friction' and is also reminiscent of the Fokker-Planck structure of the collision operator: $\mathscr{V}=\left\langle\Delta v_{\|}\right\rangle$. It is an average time rate of change of parallel velocity due to the scattering effects of collisions and accounts for the poloidal flow damping obtained from neoclassical theory. Both operators readily depend on the parallel velocity $v_{\|}$, the radial coordinate $r$ through the temperature profile $T$ and the energy $E=m v_{\|}^{2} / 2+\mu B$ through the total velocity $v^{2}=E / T$. The thermal velocity is $v_{T}=\sqrt{T / m}, m$ being the ion mass. The safety factor is $q, R_{0}$ is the major radius evaluated on the midplane and $\varepsilon=r / R$. The ion-ion collision 
frequency:

$$
v_{i i}=\frac{4 \sqrt{\pi}}{3} \frac{n e^{4} \log \Lambda}{\left(4 \pi \varepsilon_{0}\right)^{2} m^{2} v_{T}^{3}}
$$

is here expressed in terms of the dimensionless ion-ion collisionality parameter $v_{\star}$ :

$$
v_{\star}=\frac{q R_{0}}{v_{T}} \frac{v_{i i}}{\varepsilon^{3 / 2}}
$$

where $\varepsilon_{0}$ is the permittivity of free space, $\log \Lambda \approx 17$ the Coulomb logarithm and $n$ the ion density. Note that even though the collision operator Eq.(3) does depend on the energy, the advection is only performed in the parallel direction. We shall illustrate this idea in the following section, but let us just state at this point that such an operator is numerically extremely efficient since its parallelisation over velocity space $\left(v_{\|}, \mu\right)$ is intrinsic: each processor, or set of processors, has its own value for $\mu$ and performs its own collisions in the parallel direction. The explicit expressions for Eqs.(4) and (5) involve the error function $\Phi$ :

$$
\begin{aligned}
\Phi(v) & =\frac{2}{\sqrt{\pi}} \int_{0}^{v} e^{-x^{2}} \mathrm{dx} \\
\Phi^{\prime}(v) & =\frac{2}{\sqrt{\pi}} e^{-v^{2}}
\end{aligned}
$$

and the Chandrasekhar function $G$ :

$$
G(v)=\frac{\Phi(v)-v \Phi^{\prime}(v)}{2 v^{2}}
$$

Using Eq.(5), one can trivially see that the solution for the $f u l l-f$ collisional problem is the local Maxwellian $f_{L M}=n /(2 \pi T / m)^{3 / 2} \exp (-E / T)$ for both the bulk equilibrium and the fluctuations, which allows one to write the particle-conserving collision operator Eq.(3) in the compact form:

$$
\mathscr{C}(f)=\partial_{v_{\|}}\left\{\mathscr{D} f_{M} \partial_{v_{\|}}\left(\frac{f}{f_{M}}\right)\right\}
$$

$\mathscr{D}$ still being given by Eq.(4). Such an operator pertains to the wider class of socalled Lorentz operators which have been extensively used in neoclassical theory. The following section aims at discussing the physics this minimal model can address before assessing its accuracy for computing the actual transport.

\section{DISCUSSION: WHICH PHYSICS CAN WE ADDRESS ?}

This point is enlightening to be discussed in the framework of the fluid theory since it proposes a simple, yet considerable insight into plasma behaviour. The momentum flux conservation reads:

$$
m n \mathrm{~d}_{t} \boldsymbol{V}_{i}=e n\left(\boldsymbol{E}+\boldsymbol{V}_{i} \times \boldsymbol{B}\right)-\boldsymbol{\nabla} p_{i}-\boldsymbol{\nabla} \cdot \overline{\overline{\mathbf{\Pi}}}_{N C}-m n v_{i e}\left(\boldsymbol{V}_{i}-\boldsymbol{V}_{e}\right)
$$


Here, fluid quantities are written in capital letters, conversely to kinetic quantities. The electron-related quantities are labelled by subscript ' $e$ ', ion quantities by subscript ' $i$ ' and $\overline{\overline{\boldsymbol{\Pi}}}_{N C}=\int \mathrm{d}^{3} \boldsymbol{v} m\left(\boldsymbol{v} \boldsymbol{v}-v^{2} / 3 \overline{\overline{\boldsymbol{I}}}\right) f$ denotes the viscous stress tensor, crucial in toroidal geometry and which reduces at leading order to the parallel viscous stress. Focussing on the poloidal angle-independent part of the latter equation, its parallel projection, at equilibrium, simply reads:

$$
e n E_{\|}-m n \mu\left(V_{\theta i}-K_{1} \frac{\nabla T B_{\varphi}}{e\left\langle B^{2}\right\rangle}\right)-m n v_{i e}\left(V_{\| i}-V_{\| e}\right)=0
$$

where $\langle\cdot\rangle$ denotes an average over flux surfaces, $B_{\varphi}$ is the toroidal component of the magnetic field and $\left\langle\boldsymbol{b} \cdot \boldsymbol{\nabla} \cdot \overline{\overline{\boldsymbol{\Pi}}}_{N C}\right\rangle_{\theta}=m n \mu\left(V_{\theta i}-K_{1} \nabla T B_{\varphi} / e\left\langle B^{2}\right\rangle\right)$. Let us first concentrate on this term. Here, $\mu$ is the neoclassical ion viscous damping frequency, which can be approximated in the low-collisionality banana and plateau regimes [16] by: $\mu \approx$ $0.78 \sqrt{\varepsilon} v_{i i} /\left(1+0.44 v_{\star}\right)$. We adopt the notation used in [17] for the proportionality quantity $K_{1}$. Its sign is predicted to depend on the collisionality regime. This result has a practical relevance when confronting e.g. to $\mathrm{L}-\mathrm{H}$ transition theories since poloidal rotation is increasingly thought to play at crucial role in the onset of high confinement regimes. Moreover, experimental measurements of the ion poloidal velocity have been performed over the years at and after the transition [4]. Practically, one correctly gets the poloidal flow damping as soon as the viscous stress tensor is correctly modelled. Physically, it means that $(i)$ magnetic pumping due to the poloidal inhomogeneity of the magnetic field and (ii) Coulomb collisional interaction between trapped and passing particles are correctly being described. Both effects combine to enslave the ion poloidal velocity on the temperature gradient, or equivalently, on the ion diamagnetic velocity. This physics is an essential part of neoclassical theory and is intrinsically embedded in this model since this model operator Eq.(11) allows to exactly recover the neoclassical viscous stress tensor in the banana and plateau regimes as demonstrated in [10].

Moving to the last term in Eq.(13), attention is drawn to the momentum conservation properties of the collision operator. This friction term accounts for the inter species electron-ion transfer of momentum. Since momentum conservation between species is central in neoclassical theory, the implementation of the conventional neoclassical equilibrium is delicate while the electron response remains adiabatic. In this case, this inter species friction needs to be replaced by a like-species friction: $m n v_{i i} V_{\| i}$, which tends to drive the distribution function towards a Maxwellian. Provided Galilean invariance is allowed, i.e. the solution of the collisional problem is a shifted Maxwellian: $f_{S M}\left(v_{\|}\right)=f_{L M}\left(v_{\|}-V_{\| i}\right)$, the correct amount of parallel friction forces is accounted for. In this spirit, a slightly more complicated collision operator as compared to Eq.(11) is constructed in [10] which satisfies both momentum and energy conservation and, from a practical point of view, consists in performing the collisions locally, in the rest frame of the moving ions. It also means that when running a gyrokinetic code, $V_{\| i}$ and $T$ must be calculated at each time step, and fuelled back in the collision operator. Differently speaking, operators without momentum conservation create an artificial friction force and overestimate the momentum loss of circulation ions and electrons which is especially important in the banana regime. Nonetheless, as clearly shown from [20], satisfying Galilean invariance is important not to $(i)$ overestimate the particle fluxes 
and (ii) underestimate the bootstrap current. Conversely, (iii) it has almost no influence on the heat transport. Bootstrap current cannot be modelled with adiabatic electrons and since neoclassical theory is ambipolar, no particle flux is allowed with a single species collisions, which we accurately verify in GYSELA. So in the perspective of studying at the same time and with the same code both neoclassical theory and turbulence, this latter neoclassical friction is not relevant since overwhelmingly dominated by the turbulent friction. Therefore, we adopt a different approach, appropriate to an adiabatic electron response. The transverse equilibrium of Eq.(12) -the classical force balance equation:

$$
E_{r}-v_{\varphi} B_{\theta}+v_{\theta} B_{\varphi}=\frac{\nabla p}{n e}
$$

connects the radial electric field and the plasma rotation with the thermodynamic forces. Since in neoclassical theory, collisions tie poloidal rotation to the temperature gradient, the neoclassical equilibrium prediction is therefore on the sum $E_{r}-v_{\varphi} B_{\theta}$ which is degenerate without a controlled source of toroidal momentum. At this stage, we do not wish to investigate the effects of toroidal rotation on neoclassical equilibrium and seek to relax this degeneracy. The accurate and self-consistent calculation of the electric field is a known central problem for a full $-f$ electrostatic gyrokinetic code [9] and therefore the restriction of conventional neoclassical equilibrium to its sub-class associated with a vanishing mean toroidal velocity allows to unambiguously relate the radial electric field to the poloidal rotation and the thermodynamic forces while modelling the exact transport as more general Galilean-invariant Fokker-Planck operators would. Differently speaking, this operator addresses a sub-class of neoclassical equilibria associated with a mean vanishing toroidal rotation, yet modelling the exact poloidal rotation and heat transport in the banana and plateau regimes. On the other hand, this collision operator is not suitable for addressing problems such as the toroidal momentum generation since it impedes the spin-up by overdamping rotation in the toroidal direction.

Another crucial point which makes collisions especially important rests with the idea that collisions alone can provide a linear damping mechanism on the mean and zonal parts of the axisymmetric $\phi_{00}$ flows. By this means, ion-ion collisions have been reported to crucially impact the level of ITG turbulent transport in zonal flow dominated regimes [18], which also corresponds to the expected operating regime of Iter. Therefore, a collision operator must accurately model the collisional damping rate of such flows, as predicted by Hinton and Rosenbluth [14]. As shown in [8], this effect is indeed very accurately modelled.

At last, the collisional system does intrinsically satisfy an H-theorem since the collision operator Eq.(11) is associated to an entropy extremalisation principle [10]. Due to this entropy principle, the full distribution function is guaranteed to relax towards a Maxwellian while most model operators only act on the perturbed distribution function. As such they are inappropriate when calculating the complete collisional equilibrium, including both the perturbed and the unperturbed problem. The neat positive entropy production rate is reminiscent of the dissipation processes occuring in velocity space at the interface between the trapped and the untrapped regions of phase space. As a diffusive boundary layer, this interface gets increasingly localised as $v_{\star}$ diminishes. Within it, the dynamics of the collisions is accurately modelled by a friction force between the 
trapped and the untrapped particles with a favoured direction along the magnetic field lines. These aspects are quantitatively discussed in Fig.3.

Briefly summarising this discussion, this operator Eq.(11) should very accurately describe $(i)$ the collisional damping of the mean and the zonal flows, (ii) the neoclassical heat transport processes and (iii) the plasma poloidal rotation while remaining numerically very efficient. Though it is not suited to study toroidal momentum generation, the expressions of the poloidal velocity and thermal fluxes in the banana and plateau regimes are correctly reproduced, even in extreme cases where the electron response is adiabatic.

\section{RECOVERING THE NEOCLASSICAL RESULTS}

Recovering neoclassical results can reveal subtle. So as not to generate artificial equilibrium flows which could prove detrimental for neoclassical equilibrium, it is important with full $-f$ codes to accurately compute the radial electric field [9], which we do not externally impose but self-consistently evolve. It is therefore interesting to notice that:

$$
\begin{aligned}
f_{0} & =\frac{\mathscr{N}}{\left(2 \pi T_{0} / m\right)^{3 / 2}} e^{-H / T_{0}} \\
\frac{e \phi_{0}}{T_{0}} & =-\log \left(\frac{n_{0}}{\mathscr{N}}\right)+c_{1} K_{0}+c_{2} I_{0}
\end{aligned}
$$

define the simplest non trivial equilibrium state consistent with vanishing electrostatic boundary perturbations. Here $H=m v_{\|}^{2} / 2+\mu B+e \phi_{0}$ is the hamiltonian, $\mathscr{N}$ is a constant, so is $T_{0}$ the temperature, $\phi_{0}$ is the initial (and equilibrium) electric potential depending on the radial coordinate only, $n_{0}$ is the density profile, $K_{0}$ and $I_{0}$ are the modified Bessel functions of the first type and $c_{1}$ and $c_{2}$ are complex coefficients such that $\phi_{0}$ vanishes at the radial boundaries. This special choice Eq.(15) is obviously $(i)$ a motion invariant and $(\mathrm{ii})$ satisfies $\mathscr{C}\left(f_{0}\right)=0$ since $\phi_{0}$ does not depend on the velocity. Neoclassical theory predicts $v_{\theta} \propto \nabla T / e B$, as we shall discuss in the following paragraphs, thus in the case of a constant temperature $T_{0}$, the usual force balance equation Eq.(14) trivially reduces to: $e \nabla \phi / T_{0}=-\nabla n / n$, the electric field balancing the density gradient. When initialising GYSELA with Eqs.(15) and (16), the equilibrium electric field is indeed given by Eq.(16). Also, when either starting from a local Maxwellian or a canonical Maxellian $[15,1,9]$, the equilibrium electrostatic potential $\phi$ adjusts as expected on the density gradient. With these elementary checks performed, we now investigate the general case of a non vanishing temperature gradient. In the remainder of this paper, GYSELA is now initialised with the canonical equilibrium. The problem is axisymmetric. Typical parameters at mid radius for all the following simulations read: $R_{0} / L_{T}=5$ (no unstable modes), $R_{0} / L_{n}=2$ where $L_{x}=x / \nabla x$ is the gradient length for quantity $x$, $\varepsilon=r / R=0.15$ and $q=1.4$. The typical grid size at $\rho_{\star}=\rho_{i} / a=1 / 256$ involves over $10^{9}$ grid points on a half-torus $\left(r, \theta, \phi, v_{\|}, \mu\right)=(256,256,8,128,16)$ mesh.

The electric potential then adjusts so as to satisfy Eq.(14), as illustrated in Figs.2-(a) and 2-(b). The poloidal velocity $v_{\theta}$ is displayed, calculated by two means: $(i)$ selfconsistently within GYSELA $v_{\theta}^{\mathrm{G}}$, as an output of the gyrokinetic-Poisson problem and (ii) by means of the force balance equation: $v_{\theta}^{\mathrm{FB}} B_{\varphi}=\partial_{r} \phi+\nabla p / n e+v_{\varphi} B_{\theta}$, each of the 

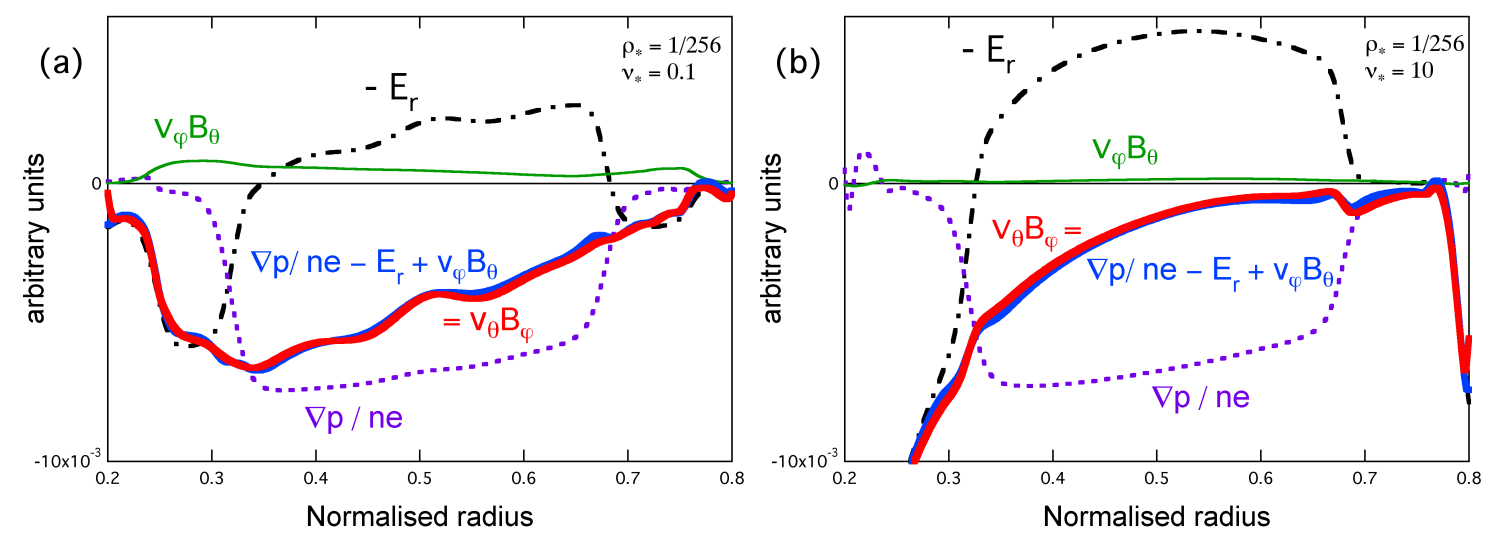

FIGURE 2. (Color online) Test of the accuracy of the force balance equation Eq.(14) in the banana (a) and the plateau (b) regimes. The poloidal velocity is evaluated by two means: either consistently evolved within GYSELA: $v_{\theta} B_{\varphi}$ or as the sum: $\nabla p / n e-E_{r}+v_{\varphi} B_{\theta}$. Excellent agreement is found, regardless of the precise moment in the simulation.

latter terms is consistently evolved withing GYSELA and constitutes an outcome of the simulation. All these quantities are here plotted. As expected from the previous section, toroidal rotation is increasingly low as the ion-ion parallel collisional friction increases from the banana regime Fig.2-(a) to the plateau regime Fig.2-(b). The response of the electric potential is therefore different in the two collisionality regimes since the poloidal velocity itself strongly depends on $v_{\star}[17,8]$. In the banana regime, the radial electric field mainly compensates the density gradient, whereas in the plateau regime, the poloidal velocity is smaller and the radial electric field increases so as to mainly compensate the pressure gradient. Hence the different behaviours observed for $E_{r}$ in Figs.2-(a) and 2-(b), while the global force balance remains satisfied with an excellent precision.

As compared to early 'classical' theories [5] in which the spatial variation of the magnetic field has no influence on the transport, neoclassical theory is deeply connected to the resonance phenomena due to particle trapping arising in an inhomogeneous magnetic field and has some crucial features on the level of collisional transport. Collisions indeed contribute to regularise the trapping singularities, broadening the highly localised region of phase space which delineates the trapped region from the untrapped. The broadening of this region with increasing collisionality is displayed in Fig.3. Due to the spatial nonuniformity of the magnetic field, iso-contours of the distribution function display the typical 'cat-eye' shape in phase space which traduces particle trapping. Starting from the canonical Maxwellian [9], the initial distribution function is a motion invariant, i.e. an exact solution of the gyrokinetic equation but not a solution of the collisional problem. It is singular at the vicinity of the loss cone, as clearly illustrated by the transverse plot of the distribution function at $\theta=0$ (red curve). The dissymmetry in $v_{\|}$comes from the choice of the canonical Maxwellian. Let us concentrate on this right-hand side of Fig.3. The two simulations at $v_{\star}=10$ and $v_{\star}=0.01$ are compared at the same time, i.e. after 10 collision times and 0.1 collision time respectively. As expected, as the collisionality increases the full distribution function $(i)$ is increasingly 


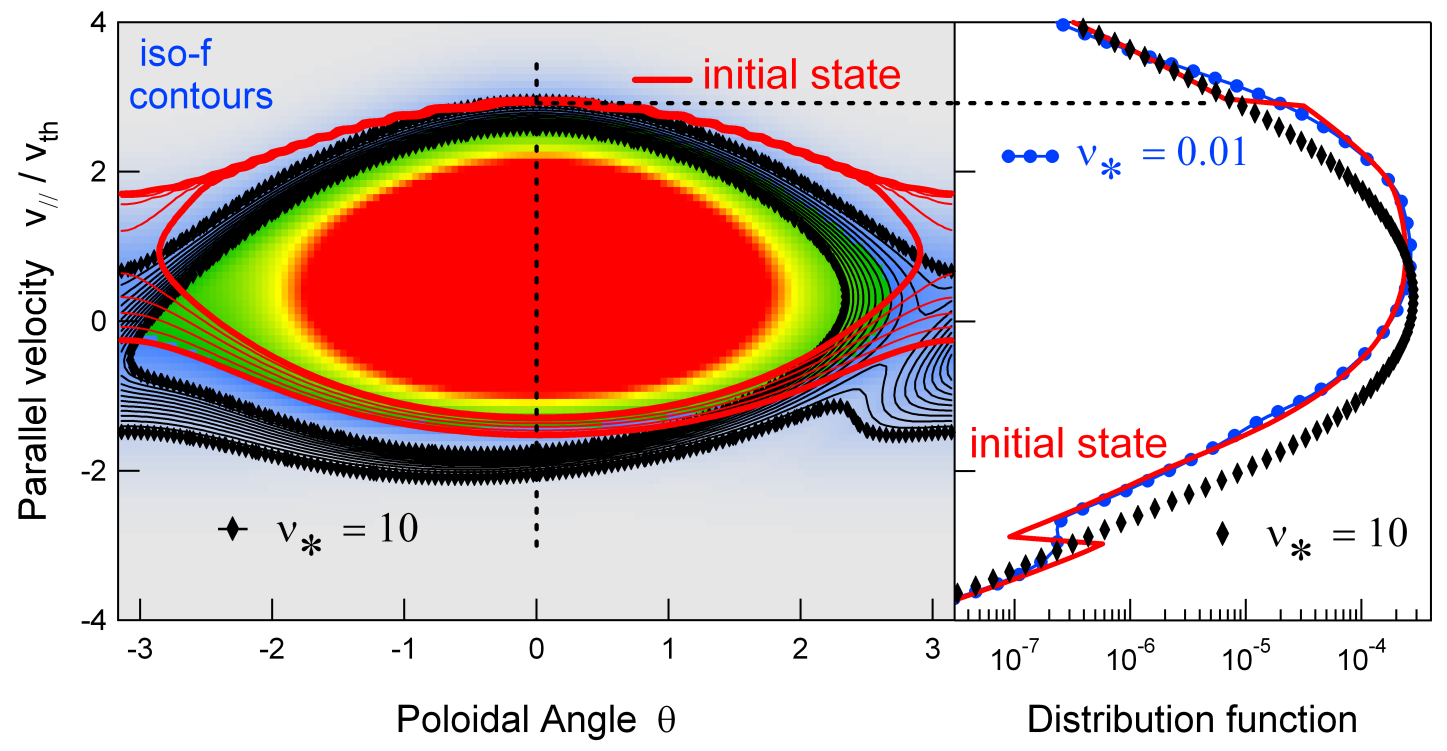

FIGURE 3. (Color online) Collisional regularisation of the full distribution function at the boundary layer between the trapped and untrapped regions of phase space. This regularisation is essentially occuring along the magnetic field lines. The island in $\left(\theta, v_{\|}\right)$space represents iso-contours of the full distribution function.

regularised and $(i i)$ relaxes towards a local Maxwellian. At low collisionalities (here in the banana regime at $v_{\star}=0.01$ ), the form of the distribution function after a few collisions is inbetween the blue and the black curves, achieving a compromise between a function of the motion invariants and a local Maxwellian. On the iso-contour plot, as best seen close to $\left(\theta, v_{\|}\right)=(0,3)$, the initial (no collision) highly-localised region delineating the trapped domain from the untrapped broadens as collisionality increases (black contours at $v_{\star}=10$ ). This regularisation occurs predominantly transversely to the island, providing a hand-waving argument why physically the correct transport could be recovered while writing the collision operator Eq.(11) with $v_{\|}$only. In the banana regime, it is well-known that this boundary layer gives the dominant contribution to the heat transport coefficient [13]. In the presence of a perturbed hamiltonian -i.e. of particle trapping, the enhancement of collisional transport, which is at the root of neoclassical transport, is due to the resonant interaction between the particles and this perturbation [10]; it dominantly occurs in this region. A correct modelisation of this boundary layer is therefore crucial to recover the correct neoclassical transport as displayed in [8].

\section{CONCLUSION}

A minimal ion-ion collision operator has been designed and successfully implemented in global and full- $f$ gyrokinetic simulations using the semi-Lagrangian GYSELA code. This operator is minimal in the sense that it reproduces the essential results of neoclassical theory while avoiding much of the parallelisation cost inherent to more general operators. This approach $(i)$ features a very efficient parallelisation with respect to the 
adiabatic variable and (ii) takes advantage of a fixed grid discretisation and as such is especially attractive in Eulerian or semi-Lagrangian-based numerical schemes.

As a special mandatory feature of full- $f$ codes, without an external forcing, the full distribution function has to relax towards a Maxwellian. This condition is not fulfilled in many simplifed operators since they were primarily designed to act on the perturbed distribution function only. Conversely, this condition is intrinsically fullfilled in the collision operator displayed here. It is especially shown that the correct neoclassical transport, poloidal rotation and mean and zonal flow damping are accurately reproduced. Building upon the advantages of the present approach, the self-consistent -and possibly significant- interplay between turbulence and collisions will be investigated in forthcoming works.

\section{ACKNOWLEDGEMENTS}

The authors want to thank Ch. Passeron for constant help in the development of the code and to acknowledge many fruitful discussions at the Festival de Théorie in Aixen-Provence, 2007. One of the authors (GDP) especially wants to acknowledge many stimulating discussions with P. H. Diamond and Z. Lin. This work, supported by the European Communities under the contract of Association between EURATOM and CEA, was carried out within the framework of the European Fusion Development Agreement. The views and opinions expressed herein do not necessarily reflect those of the European Commission.

\section{REFERENCES}

1. P. Angelino, A. Bottino, R. Hatzky, S. Jolliet, O. Sauter, T. M. Tran, and L. Villard. On the definition of a kinetic equilibrium in global gyrokinetic simulations. Physics of Plasmas, 13(5):052304, 2006.

2. R. E. Bell, F. M. Levinton, S. H. Batha, E. J. Synakowski, , and M. C. Zarnstorff. Poloidal rotation in tftr reversed shear plasmas. Phys. Rev. Lett., 81:1429, August 1998.

3. A.J. Brizard and T.S. Hahm. Foundations of nonlinear gyrokinetic theory. Reviews of Modern Physics, 79(2):421-468, 2007.

4. K. H. Burrell, E. J. Doyle, P. Gohil, R. J. Groebner, J. Kim, R. J. La Haye, L. L. Lao, R. A. Moyer, T. H. Osborne, W. A. Peebles, C. L. Rettig, T. H. Rhodes, and D. M. Thomas. Role of the radial electric field in the transition from 1 (low) mode to h (high) mode to vh (very high) mode in the diii-d tokamak. Phys. Plasmas, 1:1536, 1994.

5. S. Chapman and T. G. Cowling. The Mathematical Theory of Non-Uniform Gases. 1952.

6. K. Crombé, Y. Andrew, M. Brix, C. Giroud, S. Hacquin, N. C. Hawkes, A. Murari, M. F. F. Nave, J. Ongena, V. Parail, G. Van Oost, I. Voitsekhovitch, and K.-D. Zastrow. Poloidal rotation dynamics, radial electric field, and neoclassical theory in the jet internal-transport-barrier region. Phys. Rev. Lett., 95:155003, October 2005.

7. P. H. Diamond, S-I. Itoh, K. Itoh, and T. S. Hahm. Zonal flows in plasma -a review. Plasma Phys. Control. Fusion, 47:R35-R161, 2005.

8. G. Dif-Pradalier, V. Grandgirard, Y. Sarazin, X. Garbet, Ph. Ghendrih, and P. Angelino. Gyrokinetic simulations of neoclassical transport using a minimal collision operator. in preparation.

9. G. Dif-Pradalier, V. Grandgirard, Y. Sarazin, X. Garbet, Ph. Ghendrih, and P. Angelino. On the influence of initial state and boundary conditions on gyrokinetic turbulence. Phys. Plasmas, 15:042315, 2008.

10. X. Garbet, G. Dif-Pradalier, C. Nguyen, P. Angelino, Y. Sarazin, V. Grandgirard, P. Ghendrih, and A. Samain. A minimal collision operator for implementing neoclassical transport in gyrokinetic 
simulations. in preparation, (this conference also).

11. V. Grandgirard, M. Brunetti, P. Bertrand, N. Besse, X. Garbet, Ph. Ghendrih, G. Manfredi, Y. Sarazin, O. Sauter, E. Sonnendrucker, J. Vaclavik, and L. Villard. A drift-kinetic semi-lagrangian 4d code for ion turbulence simulation. Journal of Computational Physics, 217(2):395-423, September 2006.

12. V. Grandgirard, Y. Sarazin, P. Angelino, A. Bottino, N. Crouseilles, G. Darmet, G. Dif-Pradalier, X. Garbet, Ph. Ghendrih, S. Jolliet, G. Latu, E. Sonnendrucker, and L. Villard. Global full-f gyrokinetic simulations of plasma turbulence. Plasma Phys. Control. Fusion, 49(12B):B173-B182, 2007.

13. F. L. Hinton and R. D. Hazeltine. Theory of plasma transport in toroidal confinement systems. Rev. Mod. Phys., 48(2):239-308, April 1976.

14. F. L. Hinton and M. N. Rosenbluth. Dynamics of axisymmetric $(e \times b)$ and poloidal flows in tokamaks. Plasma Phys. Control. Fusion, 41:A653-A662, 1999.

15. Y. Idomura, S. Tokuda, and Y. Kishimoto. Global gyrokinetic simulation of ion temperature gradient driven turbulence in plasmas using a canonical maxwellian distribution. Nuclear Fusion, 43(4), April 2003.

16. Y. B. Kim, P. H. Diamond, H. Biglari, and J. D. Callen. Theory of neoclassical ion temperaturegradient-driven turbulence. Physics of Fluids B, 3(2):384-394, 1991.

17. Y. B. Kim, P. H. Diamond, and R. J. Groebner. Neoclassical poloidal and toroidal rotation in tokamaks. Phys. Fluids B, 3(8):2050, August 1991.

18. Z. Lin, T. S. Hahm, W. W. Lee, W. M. Tang, and P. H. Diamond. Effects of collisional zonal flow damping on turbulent transport. Phys. Rev. Letters, 83(18):3645, November 1999.

19. Z. Lin, T. S. Hahm, W. W. Lee, W. M. Tang, and R. B. White. Turbulent transport reduction by zonal flows: Massively parallel simulations. Science, 281:1835-1837, 1998.

20. Z. Lin, W. M. Tang, and W. W. Lee. Gyrokinetic particle simulation of neoclassical transport. Physics of Plasmas, 2(8):2975-2988, 1995.

21. R. M. McDermott, J.W. Hughes, B. Lipschultz, K. Marr, and D. Whyte. Edge radial electric field structure on alcator c-mod. US TTF, 2008.

22. M. N. Rosenbluth and F. L. Hinton. Poloidal flow driven by ion-temperature-gradient turbulence in tokamaks. Phys. Rev. Lett., 80:724-727, 1998. 